\title{
The role of numerical modelling in development of new refrigeration systems and equipment
}

\author{
B. Pavković \\ Faculty of Engineering, University of Rijeka, Croatia
}

\begin{abstract}
This paper presents trends in the application of numerical models for analysis and development of new refrigeration systems and equipment. The application of empirical, theoretical and fundamental principle algorithms and models is discussed. Features of steady-state, quasi steady-state and transient models are analysed and examples are presented. Application of system simulation programs and design programs is discussed.
\end{abstract}

Keywords: models, algorithms, numerical simulation, refrigeration.

\section{Introduction}

Number of papers dealing with numerical modelling and simulation has been increasing during past two decades due to increased computer power which makes possible faster calculations within reasonably acceptable time. In order to get the information on the present state of activities in modelling and simulation of refrigeration equipment, the search was performed for keywords "numerical, modelling and simulation" in a Fridoc database of International Institute of Refrigeration (IIR). Fridoc contains over 75.000 references concerning articles and books on all refrigeration application sectors published since 1982 and data are derived from all international literature in these fields (journals, conference proceedings, books, etc.) [1]. Results of search have shown that more than 5400 items had been published till 2005.

Distribution of total number of papers in Fridoc database for last 15 years is presented in Fig. 1, and it is compared with increase of CPU power related to the state in 1989 evaluated according to data taken from literature [2]. It is obvious that the huge increase of numerical modelling and simulation of refrigerating 
equipment or systems has been present in last 15 years, especially in last two years, and it seems to be reasonable to expect further increase. Slight decrease of published papers between 1995 and 2003 can be attributed to the fact that a majority of problems in refrigeration related to transition on new refrigerants according to the Montreal protocol (1987) was temporarily solved, and a stabilization of research activities followed. New increase of research in last two years could be connected to new energy crisis at a doorstep and to the development of system suitable for use of natural refrigerants instead of hydro fluorocarbons which exert significant global warming potential. Numerical modelling is inevitable in such a research.

Further, we can say that it does not seem realistic to expect that all papers published in the field of refrigeration are included in Fridoc database, which means that a much higher total number of papers published on that topic can be expected.

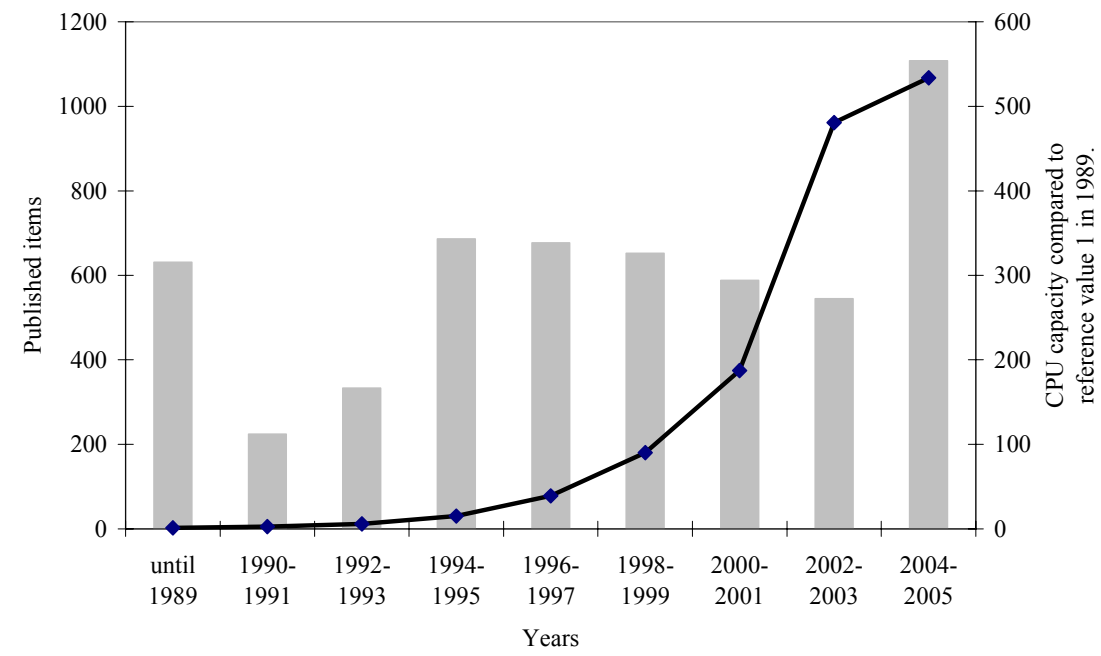

Figure 1: Total number of published items in Fridoc database including keywords "numerical, modelling, simulation" (bars) and CPU power related to CPU power in 1989 (line).

\section{Aims and objects of modelling in refrigeration research}

Refrigerating engineering includes research of heat transfer, single-phase and multi-phase fluid flow, analyses of different refrigeration cycles, research of refrigerants, mixtures of refrigerants and secondary refrigerants comprising influence of their properties on a refrigeration cycle, compressor design, optimization and application, design and optimization of heat exchangers with and without phase change of fluids, entire system and control system design etc. 
Such a wide range makes refrigerating engineering one of the most complex research fields in engineering.

Modelling can be used for research purposes in all those applications. The aims of model development are different, depending on the application area and the focus of researcher's interest. Sometimes the model is used only for energy analyses, sometimes for design or optimization of entire refrigeration system and sometimes for a single component design or optimization. It can be used also for thorough analysis of phenomena which occur in fluid flow with heat and mass transfer and which can not be analysed experimentally without a great cost of time.

Depending on aims and objects of modelling we can use steady-state, quasisteady-state and transient models.

Steady-state models are suitable for design of heat transfer equipment. Those models can be very simple, but also very complex and comprehensive, based on physical laws and including complex algorithm. The most important feature of steady-state models is that variables are not time-dependent.

A transient model is applied when at least one variable is time dependent.

A quasi-static or quasi-steady-state model is a transition from static towards transient model. At a certain time point, the system is in equilibrium. It is a combination of two or more steady-state models and it gives sufficiently accurate time average values of output values, when the user is not interested in all time variations of the output variables [3].

Application of transient models and algorithms is suitable for system simulation, where the influence of weather data, variable load and control system actions brings time-dependent perturbations. Former is the most important characteristic of all refrigeration systems.

The level of model accuracy and its volume depends on investigation's aim, and it is closely connected with processor time. We can use empirical or semiempirical "black box" models, semi-theoretical models, or as a highest level, fundamental principles based models and algorithms.

Black box models ignore physical laws and the equipment is treated as a single unit. There is no information about interactions between the components of the equipment, its dimensions, properties etc. Regression analysis, aided by knowledge of general engineering principles is used to fit the equations to discrete performance data obtained from equipment manufacturer, from laboratory tests or from more complex models. Application of the model is limited within the range of available data set used for creation of coefficients of equations achieved by regression analysis [4].

Semi-theoretical models and algorithms are physically-based, but for the reason of simplicity, calculation speed or lack of information, may use empirical expressions for evaluating coefficients, or may use the "black box" models for simulation of particular components of the system [4].

Fundamental principle (physically based) models do not use a "black box" concept. Instead, general principles of thermodynamics, heat and mass transfer and momentum transfer are used to predict temperatures, pressures, fluid mass flows and energy rates within each component of the system. Sometimes semi- 
empirical correlations are used within those models to override uncertainties and fill in gaps in theory [4]. Limitations of application are the range of applicability of the theories or correlations used in simulation and considerable amount of time necessary for simulation.

The majority of the research with application of numerical modelling is concerned with heat transfer in heat exchangers, either with or without phase change. Thermodynamic analysis of refrigeration cycles is very common application of modelling as well. Dynamic modelling of the evaporator (liquid or air cooler) performance with fully distributed fundamental principle models is one of the most demanding tasks in numerical modelling, due to the two-phase refrigerant flow problems and instabilities connected with dry-out point calculations, as well as with pressure drop calculations. Water-cooled, air-cooled and evaporative condensers are rather simple for description with transient numerical models when a refrigerant flow is through the pipe and onedimensional approach can be used. Transient models of shell-and-tube condensers with distributed parameters would involve tremendous computer capacity due to complex flow of condensing fluid inside a condenser shell. That is the reason for wide-spread bulk approach in modelling of such condensers.

Positive-displacement and centrifugal compressors are a subject of extensive research. Although a lot of dynamics is involved in compressor modelling there are a very few papers analysing that phenomena. In the simulation of whole refrigeration system, compressor dynamics includes only effects of thermal accumulation.

Black box models of expansion valves and capillary tubes are usually used as a part of chiller model. Fundamental principle models are sometimes used for capillary tubes. Development of such models for expansion valves is possible, but when the model should refer to a certain valve already produced, the modelling is often difficult due to the lack of proper information about the valve.

Another important group encompassed by modelling are liquid chillers which are widely used for air-conditioning application. Both compression and absorption chillers are described by models of different complexity and level of accuracy. Dynamic modelling with fully distributed fundamental principle models in that field is very important because it enables analyses which could not be performed experimentally, or the costs for such experiments are enormous. Using those models it is possible to analyse control strategies, optimize the heat exchangers' design and analyse operation beyond the limits of security equipment settings.

Entire refrigeration systems are also in a focus of the research interest. Those are compression as well as absorption refrigeration systems. Models of such systems are mostly produced in order to analyse energy consumption, different control strategies and system behaviour under variable operating conditions.

Besides simulation of heat exchangers with steady-state and transient models, entire refrigeration system dynamic modelling, including chiller modelling is the most important to system and equipment designer, because it gives answers about the system behaviour in real operating conditions. No matter how good and efficient components of the system are, those components will not give 
expected results if the system is not designed properly. Feedback of information from system designers and application engineers to equipment designer or producer is very important, and it is expected that modelling of the system behaviour will play an important note in design of refrigeration systems in the future.

In following sections some example of water-to-water chiller models will be presented. When we speak about chiller or heat pump, we speak about the same machine, only the control system operates in a different manner, depending on temperature level at which the heat is utilized.

\subsection{Empirical black box model: heat pump steady-state model}

Correlation for heating capacity $\dot{Q}$, cooling capacity $\dot{Q}_{e}$ and compressor's power consumption $\dot{P}_{e l}$ of a refrigeration machine can be achieved from manufacturer's data by regression. As it can be seen from the diagram at figure 2, polynomials with coefficients achieved by regression analysis are applicable only in the range covered by source data, and tremendous, even physically absurd errors can occur for values outside the range [5]. Source data can also be achieved experimentally or by using more complex dynamic heat pump models for both full and partial load. Such a model describes the steady state operation. It is very fast, but it does not take into account start-up or shutdown transients. However, temperatures changes in the heating and cooling pipelines and tanks as well as in a heated, cooled or refrigerated space are much slower than transients that take place in a heat pump during the start-up and shutdown. Therefore the influence of transients in the heat pump on temperature change in system or energy balances can be neglected in analyses concerned with the whole heating and cooling system [5]. Such a model is in a sharp contrast to the one that would be required by a heat pump designer, and which will be presented later.

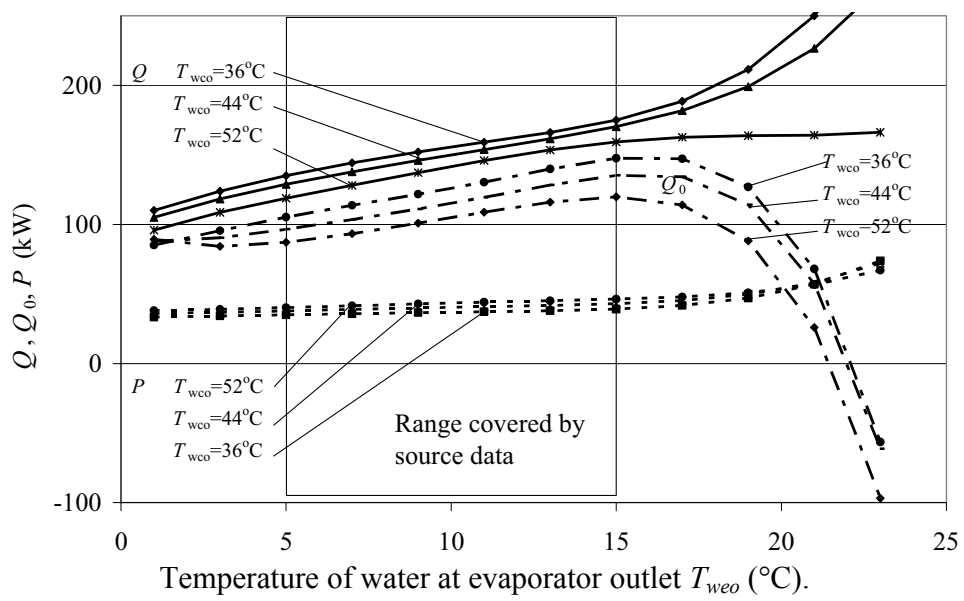

Figure 2: Example of data fit and regression polynomial for a heat pump. 


\subsection{Transient semi-empirical model of a heat pump system}

A heat pump or chiller steady-state model described in former section can be introduced as a part of a transient model representing the heating and cooling system which, besides heat pump or a chiller comprises steady-state heat exchanger models, dynamic semi-empirical lumped models of accumulation masses (heat storage tanks, boilers and pipelines) as well as models of controllers (Fig 2.) [5]. This is a dynamic, semi-empirical model, with black box models introduced for equipment, where dynamics is considered only for accumulation masses. Iterative solving for outlet temperatures is necessary for a heat pump or a chiller because its capacity depends on outlet temperatures which at the same time depend on inlet temperatures, water mass flow and heat pump capacity. For such a model, a small number of inputs appears; changing from hour to hour (time step is usually one hour). Those inputs are heat source or sink temperature, dynamic heating or cooling load, heat transfer fluid mass flows and equipment properties introduced through models of equipment. There are also a small number of outputs that will respond to these inputs. Using such a model it is possible to evaluate energy consumption or number of start-ups and shut-downs, to perform analyses of proper storage tank size, heat pump capacity, pumps' capacities, controllers' settings etc.

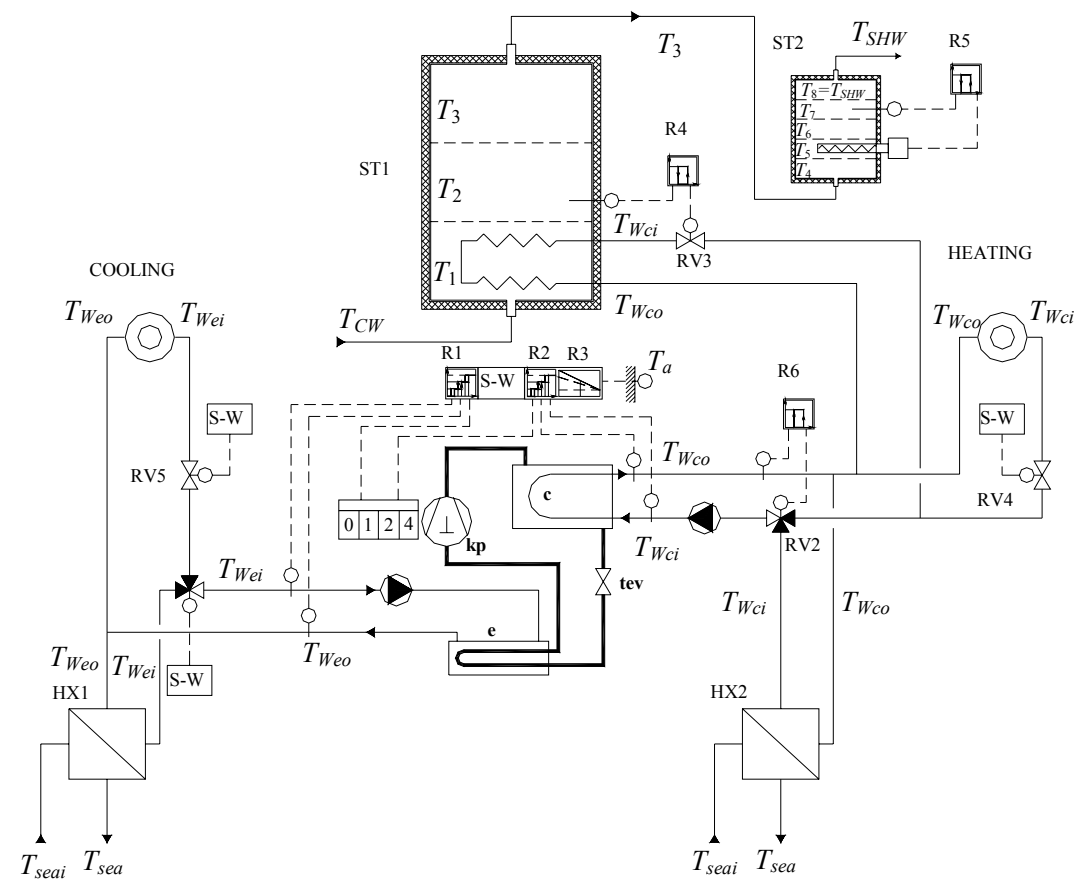

Figure 3: Heat pump heating and cooling system with description of model components and variables. 


\subsection{Fundamental principle based transient heat pump model}

Models of this kind can be used by designers of refrigerating equipment such as chillers or heat pumps, but also for design analysis of other tailor-made refrigeration systems. In such models, general principles of thermodynamics, heat transfer and fluid flow are applied to particular component of the equipment with defined properties and geometry. Those models give a designer possibility to research transient behaviour of the system during the design phase. They include a limited number of semi-empirical correlations. Such models simulate the performance of all refrigeration system components: compressors, evaporators, condensers and throttling devices and merge those together using a set of initial conditions and arbitrarily chosen, time-dependent boundary conditions. A short description of this kind of model is given with the aim to show the complexity of the problem [6].

Time-dependent boundary conditions for a heat pump model (Fig. 4) are water mass flow through the evaporator $\dot{M}_{W e}$ and condenser $\dot{M}_{W c}$, evaporator inlet water temperature $T_{W e i}$, condenser inlet water temperature $T_{W c i}$ and the action of a capacity control system.

A complex set of data, such as structure description of components and heat pump, design data about compressor, evaporator, condenser and thermo expansion valve as well as about the refrigerant properties are needed.

At the calculation start, initial distribution of refrigerant in the entire heat pump is evaluated. The dynamic calculation follows. The compressor time step is variable, depending on valve dynamics, while the time step for condenser, evaporator and expansion valve could be longer, e.g. one degree of the compressor crankshaft angle. Compressor subroutine gives suction and discharge pressures $p_{R e o}$ and $p_{R c}$ as well as compressor inlet and outlet mass flows of refrigerant $\dot{M}_{R e o}$ and $\dot{M}_{R c i}$, which are boundary conditions for evaporator and condenser. Evaporator and expansion valve subroutines have the same boundary conditions for $p_{R c}$ and $p_{R e o}$. For the refrigerant leaving the expansion valve and entering the evaporator, pressure and mass flow are determined, taking into account the pressure drop. This means that the evaporator and expansion valve are solved simultaneously, and iterations proceed until a total pressure drop across expansion valve and evaporator becomes equal to the pressure difference between $p_{R c}$ and $p_{\text {Reo }}$. In that case, the conservation equations are satisfied for the entire heat pump.

The results of the calculation are dynamic changes of pressures, temperatures and densities in the evaporator, compressor cylinders and condenser. Refrigerant distribution throughout the entire heat pump is calculated as well, which facilitates transient analysis of refrigerant loss. Compressor motor consumed power $\dot{P}_{e l}$ is calculated, while condenser and evaporator heat rates are evaluated with known water mass flows $\dot{M}_{W c}$ and $\dot{M}_{W e}$, water inlet boundary temperatures $T_{W c i}$ and $T_{W e i}$ and calculated values for outlet water temperatures $T_{W c o}$ and $T_{W e o}$. 


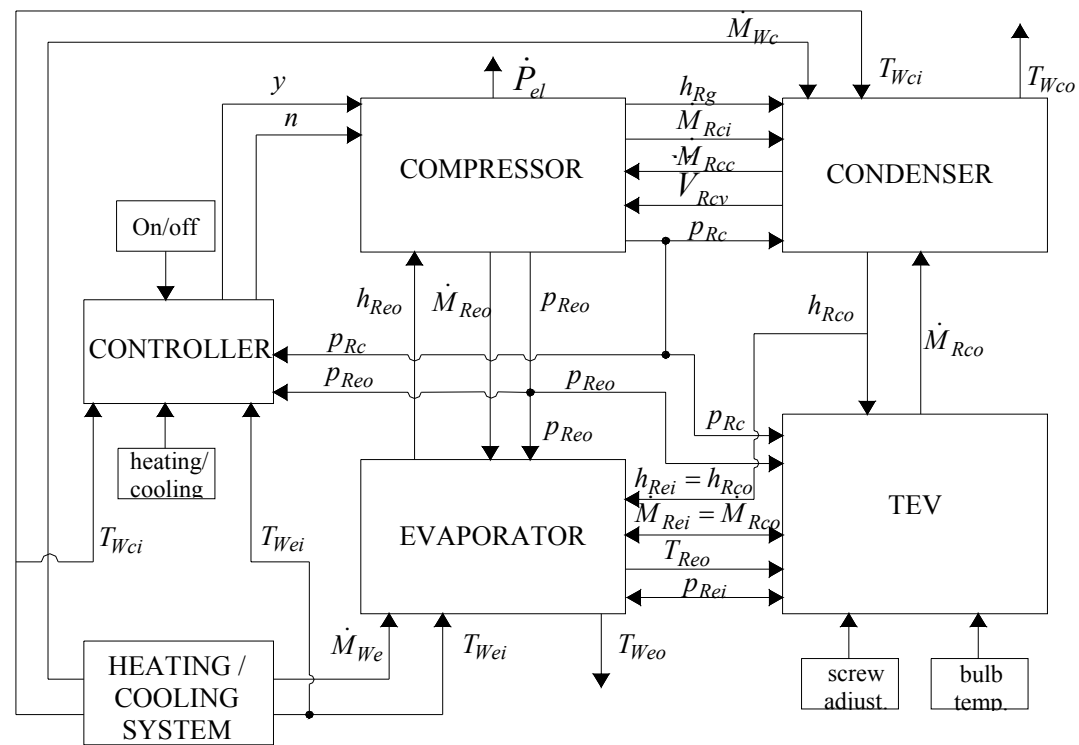

Figure 4: Data flow for a heat pump model.

The compressor model within the heat pump model is also very complex. It describes a multi-cylinder reciprocating compressor. It is based on the mathematical description of the motion of crankshaft mechanism, mass and energy conservation equations for refrigerant in a cylinder and equations for refrigerant flow through cylinder valves and valve dynamics (Fig. 5). Heat exchange between refrigerant and cylinder wall as well as between electric motor and refrigerant can also be considered.
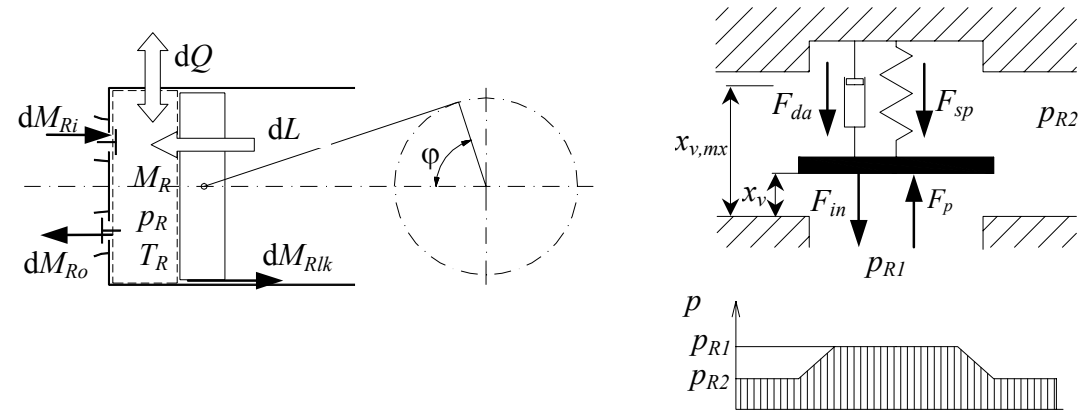

Figure 5: Compressor model-mass and energy balance for a cylinder (left) and force balance on a valve plate (right). 
Refrigerant mass flow between control volumes is driven by pressure difference. The valve cross flow area is determined from the differential equation resulting from the force balance on a valve plate (Fig. 5). The vector of volume integers and matrixes of connections control the generation and solving of the equation system during the simulation.

The evaporator model is a fully distributed parameters one. Conservation equations for mass, momentum, and energy for the refrigerant, water and pipe wall are applied. The refrigerant flow is considered one-dimensional. A bulk approach is accepted for a single control volume. The void fraction model is used to account for slip effects. Potential and kinetic energy, kinetic energy of pressure waves, the work associated with rate of pressure change as well as heat conduction in refrigerant are neglected. The effect of viscous dissipation is taken into account by pressure drop calculation, which is performed separately.

The finite volume method is used to solve the system of conservation equations.

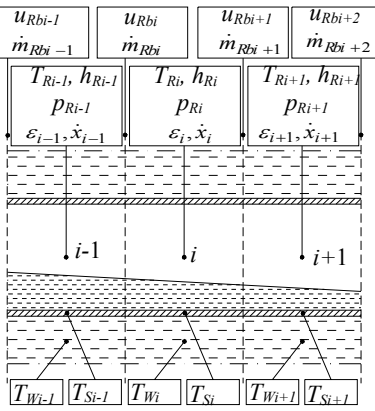

$\Delta x$

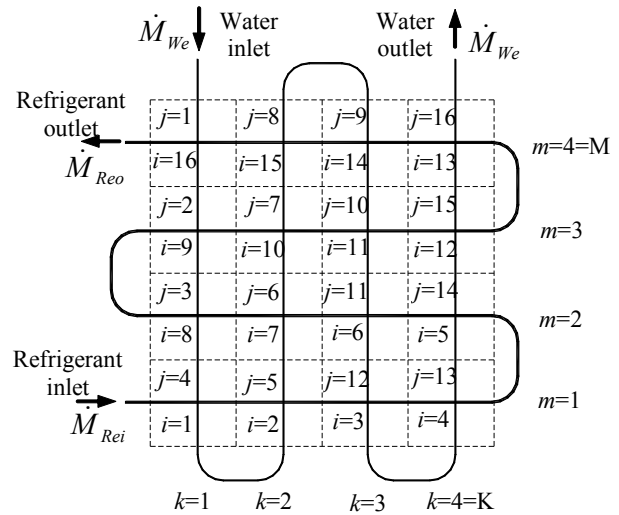

Figure 6: Evaporator model-control volumes and variables (left) and arrangement of water and refrigerant control volumes for a shell and tube evaporator (right).

The condenser model describes a water-cooled shell and tube condenser and a bulk model appears to be the most suitable one. The set of conservation equations is solved in explicit manner which can result in numerically caused errors for a case of large time steps. Therefore the inlet refrigerant mass flow $\dot{M}_{R g i}$, which depends on condenser pressure, is calculated in the compressor model which has very short time steps. The mass flow of sub cooled liquid at the bottom of the condenser $\dot{M}_{R c o}$ depends on pressure difference between the condenser and evaporator inlet, and the action of the thermostatic expansion valve (TEV).

Discretized mass and energy conservation equations for the condenser are determined from mass and energy balances for control volumes of the condenser (Figure 6). These control volumes are refrigerant vapour (1.1 and 1.2), refrigerant liquid on pipes $(2.1)$, sub cooled liquid refrigerant on the shell bottom 
(2.2), water in pipes (6.1 and 6.2) and water in deviating chambers (7.1, 7.2 and 7.3), pipes (3.1 and 3.2), plates (4.1, 4.2 and 4.3), shell in contact with refrigerant liquid (5.1) and shell in contact with refrigerant vapour (5.2).
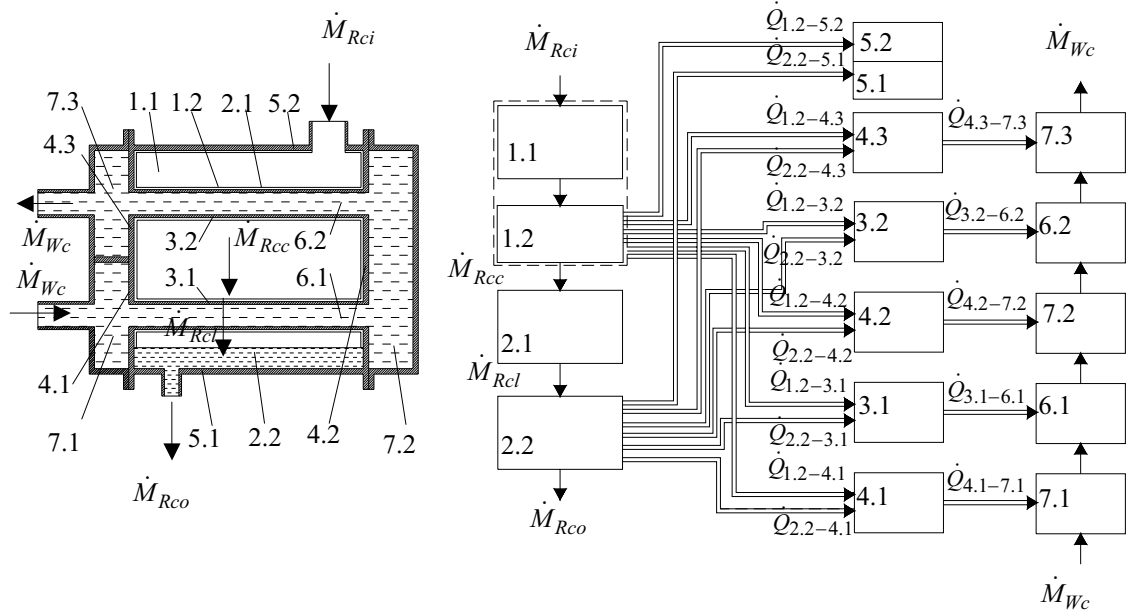

Figure 7: Control volumes and heat and mass paths for condenser model.

The thermostatic expansion valve black box model is used when the lack of information exists. Valve needle displacement and refrigerant cross flow area can be described by polynomials, with coefficients obtained from experimental data. The refrigerant mass flow through the valve is then calculated with known pressure difference. Bulb temperature transient can be taken into account through the exponential function, using the bulb time constant, which can also be determined experimentally for an observed valve.

Another type of thermostatic expansion valve model can be physically based, using equations for the equilibrium of forces acting on a valve bellow similar to equations applied for the compressor valve. Differential equations describing the heat transfer from evaporator outlet pipe to valve bulb are also necessary. In that case a set of information is needed that is often considered as a factory secret by valve producers.

\section{Algorithms and software}

A model focuses on physical laws governing the process or a device which is to be simulated and not on the computational steps in simulation. A model is developed by analysis from physical laws and empirical relationships, or it can be deduced from the experiment. Algorithm gives a computational technique for the solution of the problem stated in a model. Algorithm starts with a set of input parameters and variables and it produces a set of output variables. It has a specific range of parameter and variable values for which it is applicable. The algorithm can be expressed in mathematical notation, in graphical representation 
such as a flow chart, or in pseudo code, which follows the general format of common programming languages [3].

There is an overwhelming variety of different simulation programmes, suitable for simulations in refrigeration engineering. Majority of authors use selfdeveloped codes written in some of well known programming languages, such as FORTRAN or $\mathrm{C}++$. Those programs could be applied for computational fluid dynamics (CFD) in solving particular fluid flow and heat transfer problems in refrigeration, as well as for simulation of refrigeration components and systems. Unification of such different approaches is an uneasy task. In papers presenting the research result, it is very difficult to find the full explanation of the modelling process, or listings of computer programs. Few authors allow insight into program codes, which is easy to understand, but that lack of information also seems to be the major obstacle in unification of component models and unified approach to system modelling. Free exchange of information could conduce to considerable progress in modelling. Some commercial software packages seem to have potential to be a good basis for such a knowledge-exchange in a dynamic simulation of refrigeration systems.

TRNSYS, developed at University of Wisconsin, could be suitable for that task. TRNSYS is a transient systems simulation program with a modular structure. It recognizes a system description language in which the user specifies the components that constitute the system and the manner in which they are connected. The TRNSYS library includes many of the components. The modular nature of TRNSYS gives the program flexibility and facilitates the addition to the program of mathematical models not included in the standard TRNSYS library. Open model libraries containing some of equipment used in refrigeration engineering are available for this program [7].

HYSYS Dynamics ${ }^{\mathrm{TM}}$ is another one program of that type, produced by AspenTech, which is a modelling tool for simulation, design, performance monitoring and optimization in petroleum industries. Users may optionally link to rigorous heat exchanger design and rating tools, which make a part of Aspen HTFS $^{\text {TM }}$ family of heat exchanger software, such as TASC ${ }^{\text {TM }}$ (shell and tube exchangers), MUSE ${ }^{\mathrm{TM}}$ (multi-pass exchangers) and ACOL ${ }^{\mathrm{TM}}$ (air coolers). This provides users with more rigour when needed without leaving the HYSYS environment [8].

Some design programs for refrigeration systems and components are also available as commercial software. One typical representative is the "Refrigeration software" developed at University in Skopje, which is not a selection software but it is oriented to system and components' design. Detailed design and analysis of heat exchangers can also be performed by Aspen HTFS ${ }^{\mathrm{TM}}$ software, supported by a wide HTFS Research Network ${ }^{\mathrm{TM}}$.

When solving fluid flow and heat transfer problems within refrigeration equipment, commercial CFD programmes such as FLUENT, FLUIDYNE, ANSYS CFX, STAR-CD, EFD.Flexx, FLOWNEX etc. are the most suitable, but very important and hard to solve issue is the connection of those softwares with detailed, fundamental principle based software for equipment simulation. 


\section{Conclusion}

The increasing interest for numerical modelling in refrigeration engineering which is obvious during last few years represents a challenge for scientists and engineers concerned with refrigeration problems. Adjusting and reconsidering existing models is necessary from the viewpoint of refinement of their aims, better documentation and transparency and rearrangement of physically-based static models for application within dynamic system models. A common accepted basis for dynamic modelling should be agreed within the community of HVAC\&R scientists and engineers in order to enable the knowledge exchange and thus increased progress in dynamic modelling. Integration tools for different softwares are also a high priority task, which will enable parallel calculations with different softwares which could enable utilization of research results of different academic and industrial resources.

\section{References}

[1] http://www.iifiir.org/1enfridoc.php

[2] Patterson, D.A., Hennessy, J.L.: Computer Organisation And Design: The Hardware / Software Interface, Morgan Kaufmann Pub., USA, 2004.

[3] Bourdouxhe, J-P., Grodent, M., Lebrun, J.: Reference Guide for Dynamic Models of HVAC Equipment, ASHRAE, Atlanta GA, 1998.

[4] Yuill, G.K. et al.: An Annotated Guide to Models and Algorithms for Energy Calculations Relating to HVAC Equipment, ASHRAE, Atlanta GA, 1990.

[5] Pavković, B: Numerical Simulation of Heating and Cooling System with a Heat Pump, Proc. $17^{\text {th }}$ International Congress Energy and Environment, Opatija 2000., Croatian Solar Energy Association, Vol II, pp. 183-196., Rijeka 2000.

[6] Pavković, B., Viličić, I.: A Numerical and Experimental Investigation of the Dynamic Behavior of a Heat Pump, Proc. $10^{\text {th }}$ International Conference on Computational methods and Experimental Measurements, Alicante 2001, WIT Press Southampton, pp. 679-690., Southampton UK, 2001.

[7] http://sel.me.wisc.edu/trnsys/

[8] http://www.aspentech.com 